\title{
Image Segmentation based on Fuzzy Genetic Algorithm
}

\author{
U.Sesadri $^{\# 1}$, C.Nagaraju ${ }^{* 2}$ \\ ${ }^{\# 1}$ Research Scholar, Department of Computer Applications VTU, Belgaum ,Karnataka,India. \\ ${ }^{1}$ seshadri.madhavi@gmail.com \\ ${ }^{2}$ C.Nagaraju, Assoc.Professor, YSRCE of YVU, Proddatur,Kadapa(dist), Andrapradesh, India \\ Senior IEEE member 41513558 \\ 2 nagaraju.c@yogivemanauniversity.ac.in
}

\begin{abstract}
Image segmentation is one of the most important tasks to extract information in image processing. To satisfy increasing requirement of image segmentation, a variety of segmentation methods have been developed over the past several years. Fuzzy c-means (FCM) is unsupervised segmentation technique that has been successfully applied to future analysis, clustering, and classification but the FCM and its derivative algorithms suffer from various noises in the images. In this paper a new Fuzzy Genetic (FG) algorithm is introduce. This method describes Chromosome representation, Population Initialization, Fitness function, Genetic Operators, domain of the parameters collectively for image segmentation which produced better result comparatively FCM and its derivative methods.
\end{abstract}

Keywords- Fuzzy C-mean, Fitness function, image segmentation, Genetic Operators, Domain of the parameters

\section{INTRODUCTION}

Image segmentation is one of the most important tasks in image analysis and computer vision. Image segmentation is the process of decomposing an image into meaningful parts or objects. Many applications such as object identification, feature extraction, and object position identifications and classification requires accurate image segmentation.FCM algorithm classifies the images by grouping similar data points. It suffers from various types of noises to overcome this Fuzzy Genetic (FG) algorithm proposed. In [1], a modified Hopfield neural network model for regularized image restoration is presented. This method is used in developing algorithms with sequential, decoupled; n-simultaneous modes are updated. In [2], for blurred and noisy images two approaches are used one is Cross Correlated Multi frame (CCMF) and other one is Motion Compensated Multi frame (MCMF). In [3], one dimensional test statistics by filtering techniques for low contrasted images. In [4], it provides binary and gray scale reconstruction in different image processing applications and it is useful in image filtering and segmentation tasks. In [5] texture analysis, tree structured wavelet transform provides good analytic tool and is applicable in speech processing system. In [6] - [8] Bayesian image segmentation have used maximum a posteriori (MAP) estimation \& Markov random Fields (MRF).For synthetic images, Multi Scale Random Field Model (MSRF) provides better performance and less computation. Smoothness of motion, discontinuities of motion at boundaries and uncovered areas in the segmentation, provides better results for both synthetic and real world images by using MRF technique. In [9], for a multi scale stochastic model images likelihood calculation algorithm is presented. This algorithm provides the structure of tree and it implement parallel approaches for multi scale model and provides the better result. In [10] by observing the weakness of Histogram of Gradients(HG) and Histogram of Oriented Gradients(HOG), the new technique is implemented called as Extended Histogram of Gradient(ExHoG). This technique is unable to differentiate some local structures and produce the same feature. In [11], moving object detection algorithm by Fuzzy aggregation method is used. In [12] \& [14], saliency detection method is used. Saliency detection frame work provides hierarchical representation and high quality regional and pixel wise saliency maps. Saliency tree model is not suitable for motion fields and saliency detection in videos. The relationship between saliency estimation and Markov absorption probability, it separates the salient regions from its neighborhoods. In [15], it extends the classical Uniform Gaussian Mixture Model (UGMM) to Non Uniform Gaussian Mixture Model (NGMM). In Computer vision point set matching is an important aspect. Point sets matching include the three aspects such as i) only feature similarity ii) Uniform GMM coefficients and iii) non uniform GMM coefficients. It provides good performance for large number of qualitative and quantitative comparison experiments. In [16], Difference of Gaussian (DoG) features are highly consistence. In [17], to improve the quality of the image multi level thresholding by using Fuzzy entropy technique is used. In [18], image enhancement by using Fuzzy type2 technique and it is not applicable for document type of images.

\section{EXISTING METHOD}

In image segmentation, it is essential to reduce the effect of noise. This paper is presented based on Fuzzy C-means (FCM). It performs noise reduction, noise detection and image segmentation. By using FCM, It can estimate the probability of presence of noise in each pixel and change in the effect of that pixel. To improve the image quality and performance, apply the noise reduction and get reliable image segmentation. 


\section{A. Noise reduction}

In this paper, it distinguishes noise with respect to local neighborhood of each pixel. In addition, for detection of noise, consider the neighboring pixels. The main objective of fast image segmentation method, it needs to make use of fast noise reduction method.

\section{i) Detection of the noise pixels}

To detect a noisy pixel with the help of neighboring pixel, it follows the method proposed by efficient impulse noise reduction and it defines the neighborhood of a pixel as shown in figure 1.

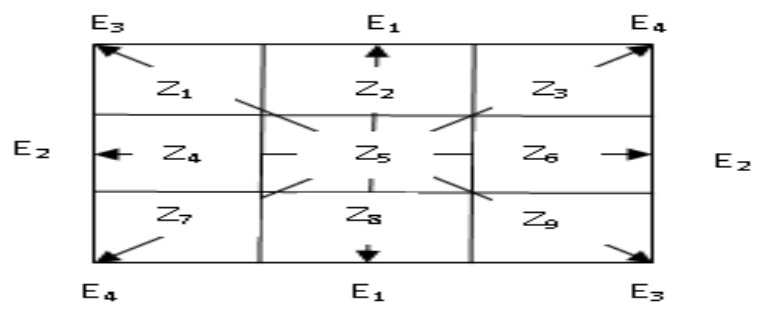

Fig 1: Matrix of 3X3 representing the coefficients and the eight edge directions.

Distance of central pixel is calculated using the following formula

Dis tan ce with NW $=\left|z_{1}-z_{5}\right|$

The neighbor pixel was obtained based on distance of the corresponding pixels. These distances were sorted in an ascending order, and 5 neighbors with longest distances are selected. If the average of these 5 values was greater than a certain threshold, it means that at least five of the neighboring pixel has been very different so that the pixel is not considered as an edge position, and it is probably a noise pixel.

\section{ii) Correction of the noisy pixels}

Neighboring pixels was corrected by identifying pixel as a noisy. The basic reason is, it uses neighboring pixel lies in the fact that a pixel is expected to be similar to its surrounding pixel. To do this, for selecting the pixel where total distance of its neighbor pixels with neighboring pixels of noisy pixel is less; this means that Euclidean distance between each neighboring pixel selected from the neighboring pixel in the same position of noisy pixel is calculated. Pixels to produce the lowest value mean that their neighbors are more like together. Two pixels with similar neighbor values are expected to be replaced y the noisy pixel.

\section{B. FCM}

FCM is an iterative clustering that produces an optimal cluster partition by minimizing the weighted group sum of squared objective function $\Theta_{\omega}$.

$$
\Theta_{\omega}=\sum_{i=1}^{\aleph} \sum_{j=1}^{\ell} v_{j i}^{\omega} \zeta^{2}\left(\chi_{i}, \vartheta_{j}\right)
$$

Where $X=\left\{\chi_{1}, \chi_{2}, \ldots, \chi_{\aleph}\right\} \subseteq \Re^{\omega}$ is the data set in the $\omega$-dimensional vector space, $\aleph$ is the number of data items, $\ell$ is the number of clusters with $2 \leq \ell<\aleph, v_{i j}$ is the degree of membership of $\chi_{i}$ in the $\mathrm{j}^{\text {th }}$ cluster, $\omega$ is the weighting exponent on each fuzzy membership, $\vartheta_{j}$ is the prototype of the center of cluster $\mathrm{j}$, $\zeta^{2}\left(\chi_{i}, \vartheta_{j}\right)$ is a Euclidean distance between object $\chi_{i}$ and cluster center $\vartheta_{j}$. A solution of the object function $\Theta_{\omega}$ can be obtained through iterative process by setting $\ell, \omega, \in$ and $v^{(0)}$ and repeat the calculation of $\vartheta_{j}^{(b)}$ and $v_{i j}$ until $\max \left\{v^{(b)}-v^{(b+1)}\right\}<\in$. The formulas for $\vartheta_{j}^{(b)}$ and $v_{i j}$ are given below.

$$
\vartheta_{j}^{(b)}=\frac{\sum_{i=1}^{\aleph}\left(v_{j i}^{(b)}\right)^{\omega} \chi_{i}}{\sum_{i=1}^{\aleph}\left(v_{j i}^{(b)}\right)^{\omega}} \text { and } v_{j i}^{(b+1)}=\frac{1}{\sum_{\omega=1}^{\ell}\left(\frac{\zeta_{j i}}{\zeta_{\omega i}}\right)^{\frac{2}{\omega-1}}}
$$




\section{PRoposed MeThoD}

The Fuzzy Genetic (FG) algorithm can be applied to work on the image enhancement. Various techniques are used to enhance the original image without losing its original properties. The following fuzzy genetic operators are used.

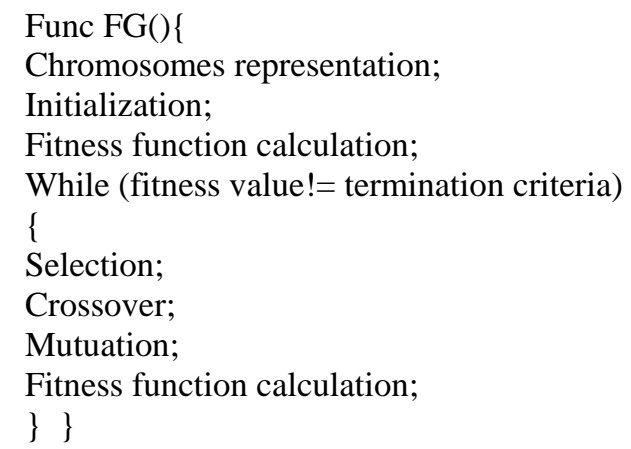

The main steps in solving a problem by using Fuzzy genetic algorithm are: 1) Chromosome representation 2) Population Initialization 3) Fitness function4) Genetic Operators like Selection, Crossover and mutation 5)Domain of the parameters

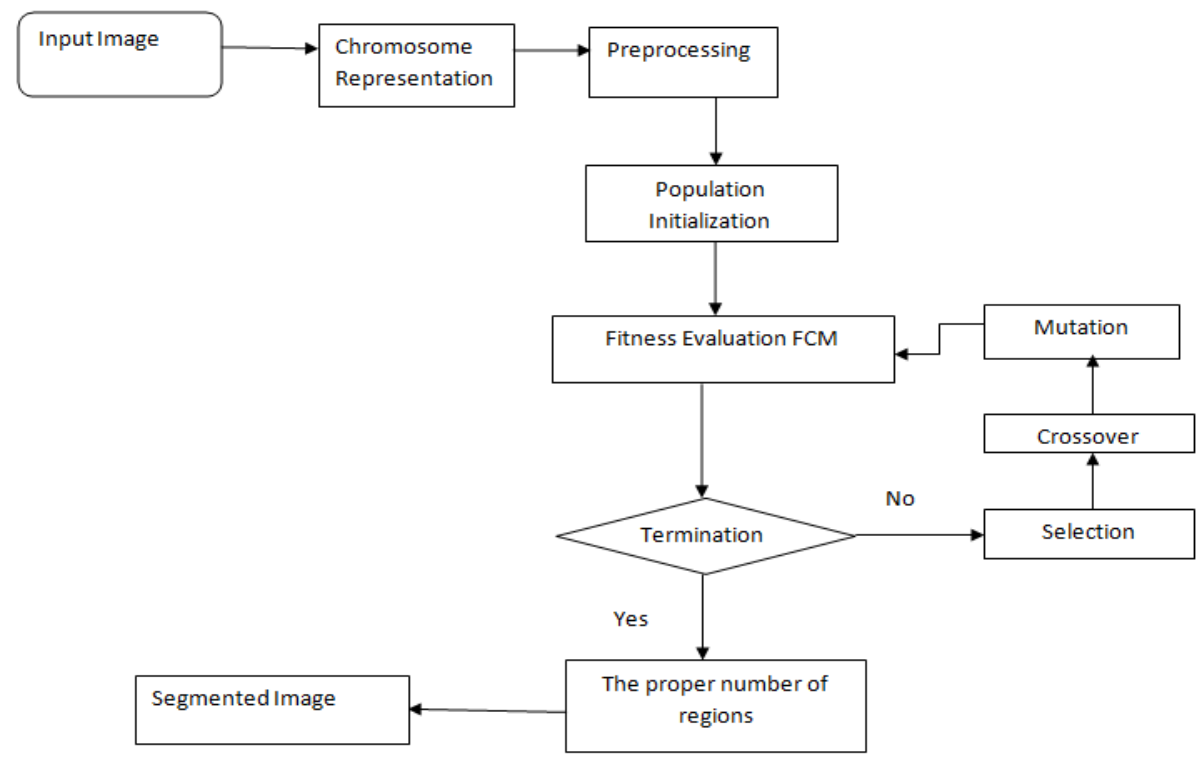

Fig 2: Fuzzy Genetic algorithm

A. Chromosome representation: The length of a chromosome shows the number of different groups of data i.e., the number of segments in an image. By measuring the length of chromosome, it controls the maximum number of segments. Chromosomes are initialized according to pixel values. Pixel values in the range 0 to 255 are used and this rage of numbers will be generated randomly for the first initialization. In table1 first row shows the chromosome values are randomly generated with length of 6 . If the chromosomes are initialized in 0 to 255 and it shows that number of segments is equal to the length of chromosome. Negative values are used; some cells of chromosomes have values that they are not used for valid centers. Suppose if it uses the negative values of cell correspond to the fact that this cell should not be considered as a data center. Hence, the number of non negative values in chromosome determines the number of data centers. Some samples of chromosomes with length 6 are given in table 1 .

TABLE 1

\begin{tabular}{|l|l|l|l|l|l|}
\hline \multicolumn{7}{|c|}{ Random generation of chromosomes } \\
\hline 30.7 & 32 & 82.8 & 130 & 188 & 163 \\
\hline-171 & 160.3 & -171 & 131.4 & -171 & 95.2 \\
\hline 82.1 & 72.5 & 169.7 & 186.7 & 78.3 & 214.9 \\
\hline 122.7 & -152 & -150 & 32.5 & 187.1 & 55.8 \\
\hline
\end{tabular}


The second chromosome in table 1 shows three centers with the values of 160.3, 131.4, and 95.2. In the above table $1^{\text {st }} \& 3^{\text {rd }}$ row chromosomes shows six valid centers, where as the $4^{\text {th }}$ one contains four valid centers.

B. Population initialization: To construct the initial population using the method described earlier. To do this we create arbitrary number of randomly initialized chromosomes. In GAs, the initial population consists of random strings. However, random binary strings, each of the length $\mathrm{pXq}$ (q bits for each of the parameters) can be considered as chromosomes or individuals of the initial population.

C. Fitness function: The reproduction is the process in which individual strings are copied according to their object function values. Objective function values are denoted by $\mathrm{F}$ and is called as Fitness function. In an enhanced image the fitness is measured by the sum of intensities of edges. The fitness function is evaluated by using two steps. First step, the pixels data set is clustered according to the centers encoded in the chromosomes under consideration. Let the each intensity value $x_{i}, i=1,2,3 \ldots m x n$ is assigned to cluster with center $z_{j}, j=1$, $2 \ldots \mathrm{K}$.

$$
\text { if }\left\|\mathrm{x}_{\mathrm{i}}-\mathrm{z}_{j}\right\|<\left\|x_{i}-z_{p}\right\|, p=1,2, \ldots, k \text {, and } \mathrm{p} \neq \mathrm{j}
$$

Second step replace the mean point of respective cluster by adjusting the values of cluster centers which are encoded in the chromosome. The new center $z_{i}^{*}$ for the cluster $C_{i}$ is given by

$$
z_{i}^{*}=\frac{1}{n_{i}} \sum_{x_{j} \in c_{i}} x_{j}, i=1,2, \ldots, k
$$

By the sum of Euclidean distances, the clustering metric $M$ is calculated.

$$
\begin{aligned}
& M=\sum_{i=1}^{k} M_{i}, i=1,2, \ldots, K \\
& \text { and } \\
& M=\sum_{x_{j} \in c_{i}}\left\|x_{j}-z_{i}\right\|
\end{aligned}
$$

The fitness function is defined as

$$
f=\frac{1}{M}
$$

Hence, for minimizing the metric $M$, the objective function $\mathrm{f}$ is maximized.

D. Genetic Operators: To produce the solution at each generation, Genetic algorithm uses the principle of selection. By using cross over and mutation Matting of parents are represented.

i) Selection: For selecting the next generation individuals, the selection operation is used. Reproduction is also called selection operator. In Genetic algorithm, Roulette wheel selection is most widely used technique. If the fitness of individual cluster $C_{i}$ in the population, then its probability is calculated as

$$
p_{i}=\frac{f_{i}}{\sum_{j=1}^{N} f_{i}}
$$

Where $\mathrm{N}$ is the number of individuals in the population.

ii) Crossover:To recombine the information Crossover operator is used. Crossover is a genetic parameter and it used to combine two chromosomes called as parents to produce new chromosome called as child (also called as offspring) chromosome. The child chromosome is also called as offspring. The result of Crossover provides a new chromosome may be better than original chromosomes. Child chromosome will have some properties from one parent and other properties from other parent. Suppose parent1 is 10110100 and parent2 is 10100011 and after performing the crossover the result which contains some part of parent1 and other from parent2.

iii) Mutation: After crossover, mutation is performed and it depends on the encoding and crossover. It maintains genetic diversity from one generation of a population of chromosomes to the next. Offspring are changed randomly by Mutation operation. For a binary encoding we can switch few randomly chosen bits and it changes bits from 0 to 1 or 1 to 0 .

Original offspring1 - 1101111010011100

Mutated offspring1- 1100111010011100

Original offspring2- 1001100100110100 
Mutated offspring2- 1001101100110100

E. Domain of the Parameters: To define a Genetic algorithm for a specific problem, the domains of parameters are used. They are (i) The population size i.e., it represents number of chromosomes in a population.(ii) The number of generations to be generated.(iii) Mutation rate i.e., the probability of new information added randomly.

\section{QUALITY PARAMETERS}

A.Mean: It is used to find the average gray levels of the image and is calculated as:

$$
\operatorname{mean}(\mu)=\frac{1}{P Q} \sum_{x=1}^{P} \sum_{y=1}^{Q} f(x, y)
$$

Where $\quad P$ and $Q$ are width and height of the image and $f(x, y)$ is gray value.

B. Standard deviation: the standard deviation of gray level image is calculated as follows

$$
\text { st }(\sigma)=\sqrt{\frac{1}{P Q} \sum_{x=1}^{P} \sum_{y=1}^{Q}(f(x, y)-\mu)^{2}}
$$

Where $\mathrm{P}, \mathrm{Q}$ are the width and height of the image, $\mu$ is mean of the image, $\mathrm{f}(\mathrm{x}, \mathrm{y})$ is gray level value of the image, $5 t(\theta)$ is standard deviation.

C. Jaccard Index: jaccard index is a statistic used for comparing the similarity and diversity of sample sets. It is calculated as:

$\operatorname{Jac}(P, Q)=\frac{|P \cap Q|}{|P \cup Q|}$

Where $\mathrm{P}$ and $\mathrm{Q}$ are both non -empty, we define jac $(\mathrm{P}, \mathrm{Q})=1$ and $0 \leq j a c(P, Q) \leq 1$

\section{RESULTS AND ANALYSIS}

To demonstrate the effectiveness and robustness we conduct few experiments. The proposed method Fuzzy Genetic Algorithm is tested and compare the existing FCM method 100 images data set.Binary performance is evaluated by using the Jaccard Index(JAC), mean $(\mu)$ and standard deviation $(\sigma)$. In this paper Fuzzy Genetic algorithm is used for image enhancement. The result analysis of this paper is divided into two methods subjective evaluation and experimental evaluation. In this section subjective evaluation of Fuzzy Gentic method. We compare the results of Fuzzy Genetic algorithm method with FCM method. Our porposed metod provides the better results for Lena, bird, Crow, two birds, three birds,elephant, things and wheel.The figure shows the comparision of existing and proposed methods and it also displays the original image histograms. In img7 provids the better solution for uneven illuminatios.
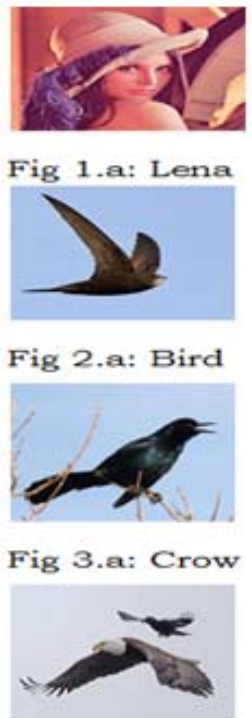

Fig 4.a: Two birds Fig 4.b
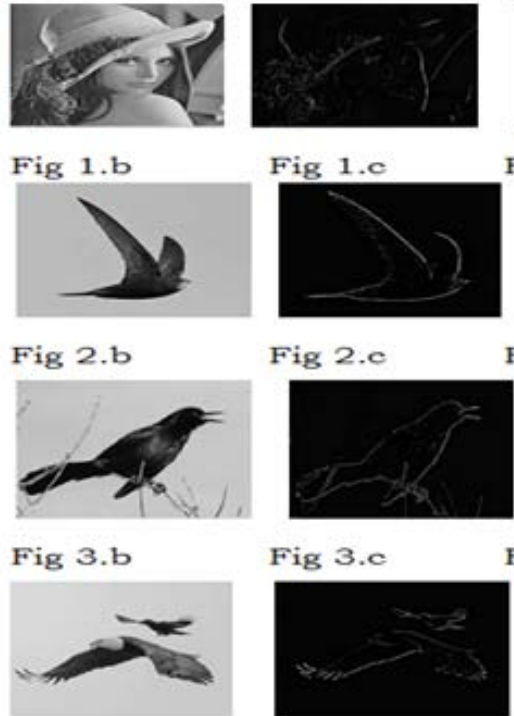

Fig 4.c
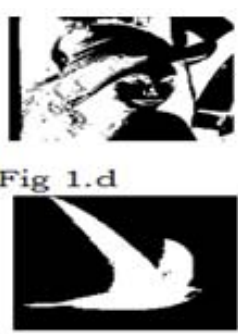

Fig 2.d
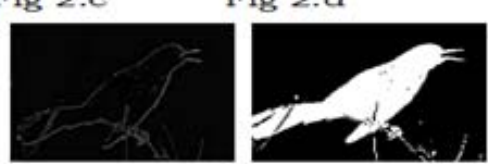

Fig 3.d

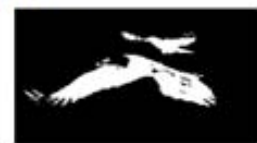

Fig 4.d
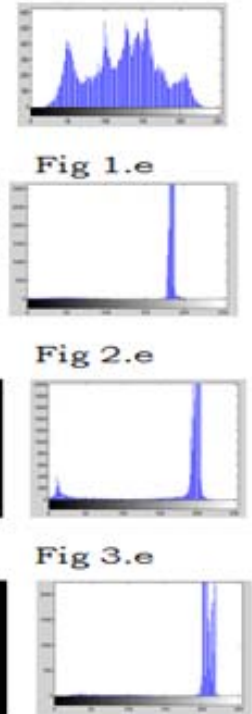

Fig $4 . e$ 

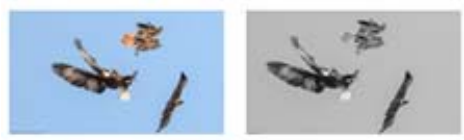

Fig 5.a: Three birds Fig 5.b
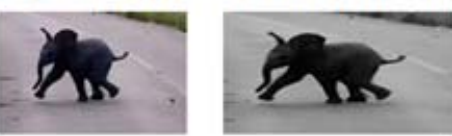

Fig 6.a: Elephant Fig 6.b
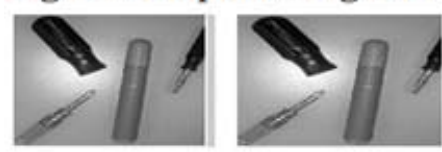

Fig 7.a: Things Fig $7 . b$

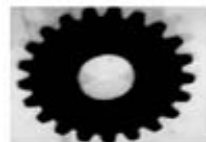

Fig 8.a: Wheel Fig 8.b
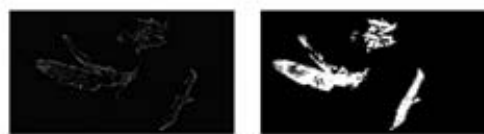

Fig 5.c

Fig 5.d
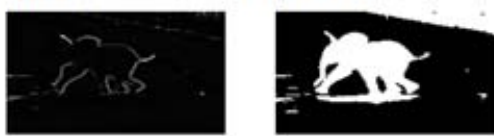

Fig 6.c

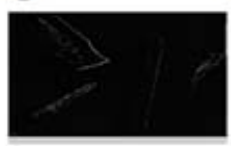

Fig 6.d

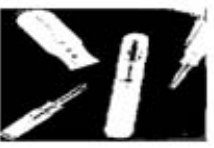

Fig 7.0

Fig $7 . d$

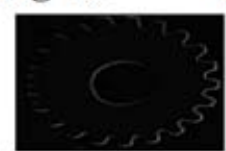

Fig 8.c

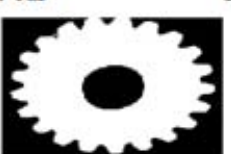

Fig 8.d

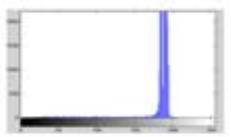

Fig 5.e

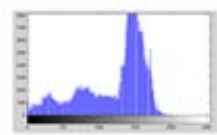

Fig 6.e

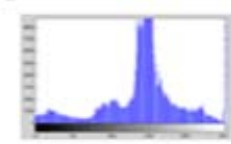

Fig $7 . e$

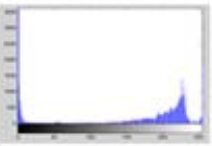

Fig $8 . e$

Fig 3 : First Colum is color image , Second Colum is gray image, third color FCM, Fourth Colum Fuzzy Genetic and Fifth Colum histogram of gray image

TABLE 2

\begin{tabular}{|l|l|l|l|}
\hline \multirow{2}{*}{ S.no } & \multicolumn{3}{|c|}{ Mean } \\
\cline { 2 - 4 } & Original image & Existing Method & Proposed Method \\
\hline 1 & Leena & 0.0170 & 0.0168 \\
\hline 2 & Bird & 0.0173 & 0.0176 \\
\hline 3 & Crow & 0.0175 & 0.0175 \\
\hline 4 & Two Birds & 0.0181 & 0.0180 \\
\hline 5 & Three Birds & 0.0183 & 0.0180 \\
\hline 6 & Elephant & 0.0168 & 0.0167 \\
\hline 7 & Four things & 0.0171 & 0.0172 \\
\hline 8 & Wheel & 0.0108 & 0.0112 \\
\hline
\end{tabular}

TABLE 3

\begin{tabular}{|l|l|l|l|}
\hline \multirow{2}{*}{ S.no } & \multicolumn{3}{|c|}{ Standard deviation } \\
\cline { 2 - 4 } & Original image & Existing Method & Proposed Method \\
\hline 1 & Leena & 0.0068 & 0.0066 \\
\hline 2 & Bird & 0.0065 & 0.0065 \\
\hline 3 & Crow & 0.0071 & 0.0070 \\
\hline 4 & Two Birds & 0.0070 & 0.0068 \\
\hline 5 & Three Birds & 0.0062 & 0.0063 \\
\hline 6 & Elephant & 0.0065 & 0.0070 \\
\hline 7 & Four things & 0.0061 & 0.0069 \\
\hline 8 & Wheel & 0.0115 & 0.0112 \\
\hline
\end{tabular}


TABLE 4

\begin{tabular}{|l|l|l|l|}
\hline \multirow{2}{*}{ S.no } & \multicolumn{3}{|c|}{ Jaccord Indexing } \\
\cline { 2 - 4 } & Original image & Existing Method & Proposed Method \\
\hline 1 & Leena & 0.0000 & 0.0201 \\
\hline 2 & Bird & 0.1831 & 0.1831 \\
\hline 3 & Crow & 0.0001 & 0.0014 \\
\hline 4 & Two Birds & 0.6104 & 0.4578 \\
\hline 5 & Three Birds & 0.0022 & 0.0007 \\
\hline 6 & Elephant & 0.0638 & 0.0384 \\
\hline 7 & Four things & 0.0649 & 0.0649 \\
\hline 8 & Wheel & 0.0017 & 0.0001 \\
\hline
\end{tabular}

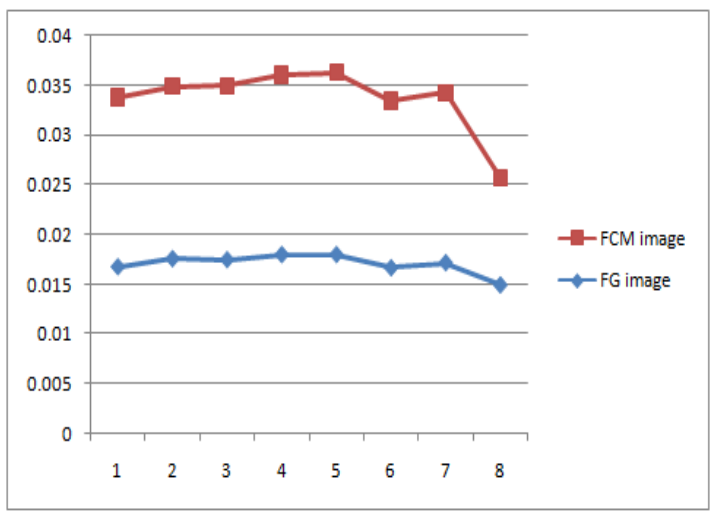

Graph 1: mean

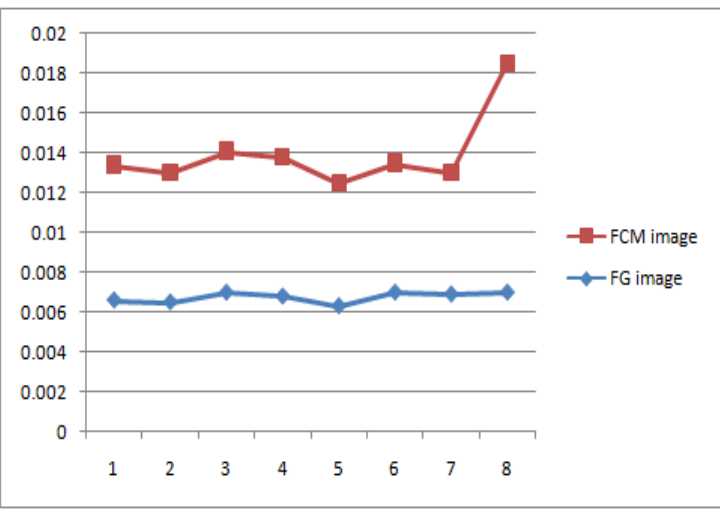

Graph 2: standard deviation

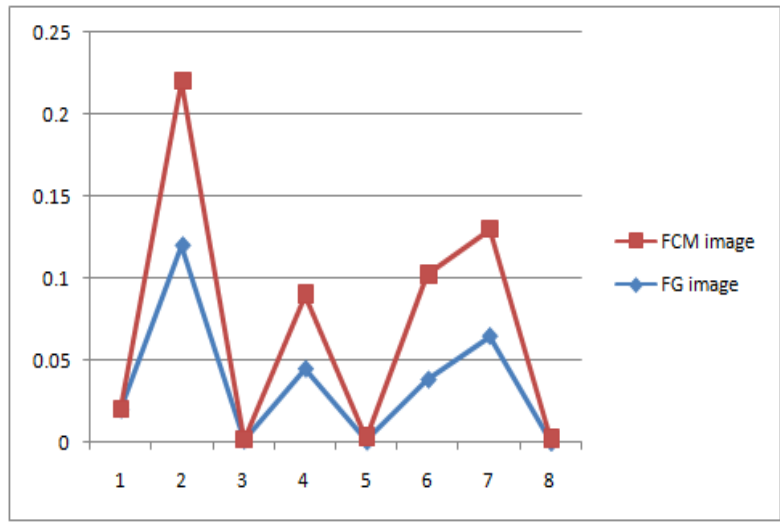

Graph 3: Jaccord indexing

\section{CONCLUSION}

In this paper new method is proposed by using Fuzzy Genetic algorithm. FCM and its derivative methods suffer from noise in the images. To overcome this drawback the proposed technique is used. It very useful for object reorganization even the image contains noisy, shadows and low contrasted images. The proposed method effectively removes shadows in image, noise in the image and identified objects even for low contrasted images. We evaluated the performance of the proposed method 3 different performance measures like mean, standard deviation and Jaccard index. This method gives better results.

\section{REFERENCES}

[1] Joon K.paik and K.Katsaggelos, “Image Restoration using a modified Hopfield Network” January 1992 journal of IEEE transactions on Image processing, Volume 1, No 1, pp 48-63

[2] Mehmet K.Ozkan, A.Tanju Erdem, M.Ibrahim sezan, “ Efficient Multiframe Wiener Restoration of Blurred and noisy image sequences, October 1992 journal of IEEE, Vol , No.4, pp 453-476.

[3] John A. Gupner, Bahaa E.ASaleh, "Image detection under low level illuminations”, Jan 1993 journal of IEEE , vol 2, No.1, PP 18-26.

[4] Luc Vincent, “ Morphological gray scale reconstruction in image analysis: Applications and efficient algorithms”, April 1993, journal of IEEE, Vol 2, No.2, pp 176-201 
[5] Tianhorng Chang and C.C Jaykuo, “Texture analysis and classification with tree structured wavelet transform”, October 1993, journal of IEEE transactions on Image processing Vol 2, No.4, pp 429-441

[6] Charles Bouman and Micael Shapiro, "Multi scale random field model for Bayesian image segmentation”, March 1994, journal of IEEE transactions on image processing, vol 3, No.2, pp 162-177.

[7] Richard R. Schultz and L Stevenson, “' A Bayesian approach to image expansion for improved definition”, May 1994, Journal of IEEE transactions on image processing vol 3. No.3 233-242.

[8] Jun Zhan, Gerald and G. Hanauer "The application of mean field theory to image motion estimation”, January 1995, Journal of IEEE transactions on image processing, Vol 4, No1, PP 19-33.

[9] Mark R. Luettgenand Alan,“ Likelihood calculation for a class of multiscale stochastic models, with application to texture discrimination”, Feb 1995, Journal of IEEE transactions on image processing Vol 4, No 2, PP 194-207

[10] "Human detection by quadratic classification on subspace of extended histogram of gradients", Jan 2014 Journal of IEEE transactions on image processing vol 23, No 1, pp 287-297

[11] Pojala CHiranjeevi and Somnath Sengupta, "Neighborhood supported model level fuzzy aggregation for moving object segmentation", Feb 2014 Journal of IEEE transactions on image processing vol 23 No 2, PP 645-658.

[12] ZhiLiu,WenbinZou and Oliver,"Saliency Tree:A Novel saliency detection framework”, May2014 IEEE transactions on image processing vol 23,No 5 PP-1997-1951.

[13] Xi Yang, Xinbo Gao and Dacheng Tao, “ AN efficient MRF embedded level set method for imafe segmentation”, Jan 2015 IEEE Transactions on image processing Vol 24 No 1, PP 9- 22.

[14] Jingang sun, Huchuan Lu and Xiuping Liu, “ Saliency region detection based on markov absorption probabilities”, May 2015 IEEE transaction on image processing Vol 24 No 5 , PP 1639-1650.

[15] Tao Wenbing and KunSun,"Robust point sets matching by fusing feature and spatial information using non uniform Gaussian mixture models”, ovember 2015 IEEE transactions on image processing Vol 24 No 11, PP 3754-1368.

[16] Soo-Chang Pei and Li-Heng Chen, ” image quality assessment using human visual DOG model fused with random forest” Nov 2015 IEEE transactions on image processing Vol 24 No 11, PP 3282-3293.

[17] U Sesadri and C. Nagaraju, "Optimal thresholding for enhancement of low contrasted images using soft computing”, Dec 2015 IEEE conference.

[18] U Sesadri and C Nagaraju, “Type 2 fuzzy soft computing technique for image enhancement”, Nov 2015 Journal of IJCSIS Vol 13 No 11, PP 106-117.

\section{AUTHORS PROFILE}

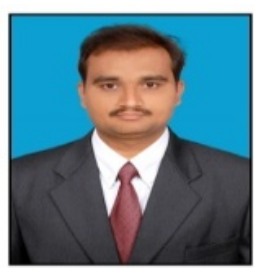

U. Sesadri, received his M.Sc. in Mathematics from SV University, Tirupati, M.E in Computer Science and Engineering from Sathyabama University, Chennai and presently pursing PhD in Digital Image Processing from V.T. University, Belgaum. He has got 8 years of teaching experience. He has attended five National Level workshops and two international level conferences. He has organized 10 National level workshops and two National level paper presentations. He has published two research journals.

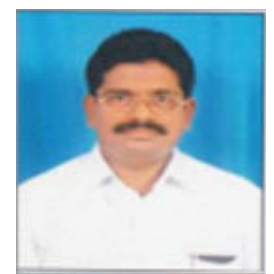

Dr. C. Naga Raju is currently working as Associate Professor and Head of the Department of Computer Science and Engineering at YSR Engineering College of Yogi vemana University, Proddatur, Kadapa District, and Andhra Pradesh, India. He received his B.TechDegree in Computer Science and Engineering from J.N.T.University, Anantapur, and M.Tech Degree in Computer Science from J.N.T.University Hyderabad and PhD in digital Image processing from J.N.T.University Hyderabad. He has got 18 years of teaching experience. He received research excellence award, teaching excellence award and Rayalaseema vidhyaratna award for his credit. He wrote text book on C \& Data structures and Pattern Recognition. He has six PhD scholars. He has published fifty six research papers in various National and International Journals and about thirty research papers in various National and International Conferences. He has attended twenty seminars and workshops. He delivered 10 keynote addresses. He is member of various professional societies like IEEE, ISTE and CSI. 